PROCEEDINGS OF THE

AMERICAN MATHEMATICAL SOCIETY

Volume 129, Number 9, Pages 2633-2642

S 0002-9939(01)05835-X

Article electronically published on February 15, 2001

\title{
STABILITY AND ALMOST PERIODICITY OF ASYMPTOTICALLY DOMINATED SEMIGROUPS OF POSITIVE OPERATORS
}

\author{
E. YU. EMEL'YANOV, U. KOHLER, F. RÄBIGER, AND M. P. H. WOLFF \\ (Communicated by Dale Alspach)
}

\begin{abstract}
We discuss conditions such that strong stability and strong asymptotic compactness of a (discrete or continuous) semiflow defined on a subset in the positive cone of an ordered Banach space is preserved under asymptotic domination. This is used to show that on a Banach lattice with order continuous norm strong stability and almost periodicity of a (discrete or strongly continuous) semigroup of positive operators is preserved under asymptotic domination.
\end{abstract}

\section{INTRODUCTION}

If $S$ and $T$ are positive linear operators on an ordered Banach space $E$ such that $S$ is dominated by $T$, then it is natural to ask which properties of the operator $T$ are inherited by the operator $S$. There are numerous results in this direction dealing with properties like compactness, weak compactness, the Dunford-Pettis property, being a kernel operator and others (see [1], 11, 22 for a survey of such results).

During the last ten years there was an increasing interest to investigate spectral and asymptotic properties of dominated operators, such as strong and uniform ergodicity, strong and uniform stability, almost periodicity, quasicompactness or certain inclusion relations among the peripheral spectra (see e.g. [2], 3], [5], [9], [10, [12, 14], [15], 16], [17, [18, 19]). Most of the results can be formulated for (powers of) operators as well as for representations of certain semigroups in the space of bounded linear operators.

In [15, Theorem 3.9] it was shown that if $T$ is a positive operator on a Banach lattice $E$ with order continuous norm such that the powers $T^{n}$ converge strongly to a projection $P_{T}$ of finite rank, then for each operator $S$ on $E$ such that $0 \leq S \leq T$ the powers $S^{n}$ are also strongly convergent. The same conclusion holds if instead of the finite rank condition on $P_{T}$ one requires that the spectrum $\sigma(T)$ of $T$ does not contain the whole unit circle (see [17, Corollary 4.3]). Analogous statements hold for the inheritance of almost periodicity under domination (see [15. Proposition 3.10], [17, Theorem 4.2]). It is open whether in the described situation any of the additional conditions on the dominating operator $T$ is needed.

Received by the editors October 29, 1998 and, in revised form, January 7, 2000.

2000 Mathematics Subject Classification. Primary 47D03, 47B65, 46B40, 46L99.

Key words and phrases. Stability, almost periodicity, semigroup representation, asymptotic domination, ordered Banach space. 
In the present paper we will answer this question. Our approach allows us to treat an even more general situation. First we show that on a set $D$ in the positive cone of a uniformly order convex Banach space strong stability and strong asymptotic compactness of a non-expansive (discrete or continuous) semiflow is preserved under asymptotic domination (Theorem 3.1 and 3.3). As a special case we obtain on $L^{1}$-spaces inheritance of strong stability and almost periodicity for (discrete and strongly continuous) semigroups of positive linear operators which are asymptotically dominated by a semigroup of positive linear contractions. From this we derive the corresponding result for semigroups on Banach lattices with order continuous norm without assuming contractivity of the asymptotically dominating semigroup (Theorem 4.3 and 4.5). An example shows that such results are no longer true if the asymptotically dominated semigroup is not positive (Example 4.7).

Our notation is standard and follows the books [11] and 20]. We recall some frequently used notions. For a Banach space $E$ let $B_{E}$ be the closed unit ball, $\mathcal{L}(E)$ the space of bounded (linear) operators on $E$, and $E^{\prime}$ the space of bounded linear forms on $E$. If $E$ is an ordered Banach space, then $E_{+}:=\{x \in E: x \geq 0\}$ is the positive cone of $E$. An operator $T$ on $E$ is called positive if $T E_{+} \subseteq E_{+}$. By $\mathcal{L}(E)_{+}$ and $E_{+}^{\prime}$ we denote the set of positive bounded operators and the set of positive linear forms on $E$, respectively. If $E$ is a Banach lattice we write $\vee$ and $\wedge$ for the lattice operations sup and inf. As usual we denote by $x_{+}, x_{-}$and $|x|$ the positive part, the negative part, and the modulus of $x \in E$. Note that with $E, E^{\prime}$ is also a Banach lattice with positive cone $E_{+}^{\prime}$. For further details on ordered Banach spaces and Banach lattices we refer to [4, [21], and [11], [20].

\section{UNIFORMLY ORDER CONVEX BANACH SPACES}

Throughout the whole section $E$ denotes an ordered Banach space with a closed, generating, normal positive cone $E_{+}$. Moreover, we assume that the norm of $E$ is monotone on $E_{+}$, i.e., if $x, y \in E$ such that $0 \leq x \leq y$, then $\|x\| \leq\|y\|$. We introduce the following class of ordered Banach spaces.

Definition 2.1. The ordered Banach space $E$ is called uniformly order convex if for every $\varepsilon>0$ there exists $\delta>0$ such that whenever $x, y \in\left(B_{E}\right)_{+}, 0 \leq x \leq y$, and $\|y\|-\|x\|<\delta$, then $\|x-y\|<\varepsilon$.

For $z \in E$ denote by $d\left(z, E_{+}\right):=\inf \left\{\|z-x\|: x \in E_{+}\right\}$the distance of $z$ to $E_{+}$. One can easily see that an ordered Banach space $E$ is uniformly order convex if and only if

$$
\begin{aligned}
& \text { for every } M>0 \text { and every } \varepsilon>0 \text { there exists } \delta>0 \text { such that } \\
& \text { whenever } x, y \in M \cdot\left(B_{E}\right)_{+}, d\left(y-x, E_{+}\right)<\delta \text {, and }|\|y\|-\|x\||<\delta, \\
& \text { then }\|y-x\|<\varepsilon \text {. }
\end{aligned}
$$

We mention the most important examples of uniformly order convex Banach spaces.

Example 2.2. a) Every ordered Banach space $E$ with uniformly convex norm is uniformly order convex (cf. [7] Section 1.e]). In particular, every $L^{p}$-space, $1<p<$ $\infty$, is uniformly order convex.

b) Every ordered Banach space $E$ whose norm is additive on $E_{+}$is uniformly order convex. In particular, this is the case for $L^{1}$-spaces, the selfadjoint part of the dual of a $C^{*}$-algebra and the selfadjoint part of the predual of a $W^{*}$-algebra. 
c) Let $E$ be a Banach lattice with $p$-superadditive norm $(1 \leq p<\infty)$, i.e., $\left(\sum_{k=1}^{n}\left\|x_{k}\right\|^{p}\right)^{1 / p} \leq\left\|\sum_{k=1}^{n} x_{k}\right\|$ for all $x_{1}, \ldots, x_{n} \in E_{+}, n \in \mathbb{N}$ (see [11, 2.8.2]). If $x, y \in\left(B_{E}\right)_{+}$and $0 \leq x \leq y$, then $\|y\| \geq\left(\|y-x\|^{p}+\|x\|^{p}\right)^{1 / p}$, and hence $\|y-x\| \leq\left(\|y\|^{p}-\|x\|^{p}\right)^{1 / p}$. In particular, we obtain that $E$ is uniformly order convex. As a consequence on every $p$-concave Banach lattice $E$ there is an equivalent lattice norm for which $E$ is uniformly order convex (see [11, 2.8.8] and [7, p.85]).

Recall that a Banach lattice $E$ is a $K B$-space if every increasing norm bounded sequence in $E_{+}$converges in norm (see [11, 2.4.11]).

Proposition 2.3. Every uniformly order convex Banach lattice $E$ is a KB-space.

Proof. Let $\left(x_{n}\right)$ be an increasing, norm bounded sequence in $E_{+}$. Then

$$
\lim _{n, m \rightarrow \infty}\left\|x_{n}\right\|-\left\|x_{m}\right\|=0 .
$$

The uniform order convexity of the space $E$ implies that $\left(x_{n}\right)$ is a Cauchy sequence in $E$, and hence $\left(x_{n}\right)$ is convergent.

Let $D \subseteq E$. A map $P: D \rightarrow E$ is called non-expansive if $\|P x\| \leq\|x\|$ for all $x \in D$. The following lemma will play a central role in the investigations of Sections 3 and 4 .

Lemma 2.4. Let $E$ be a uniformly order convex Banach space, $D \subseteq E_{+}, J=\mathbb{N}$ or $\mathbb{R}_{+},\left(x_{t}\right)_{t \in J}$ a family in $D$, and $P_{t}: D \rightarrow E_{+}, t \in J$, non-expansive mappings. Let $\left(t_{n}\right)$ be a sequence in $J$ converging to $\infty$ and assume that:

(i) $\lim _{n} P_{t_{n}-t_{m}} x_{t_{m}}$ exists for all $m \in \mathbb{N}$, and

(ii) $\lim _{n} d\left(P_{t_{n}-t_{m}} x_{t_{m}}-x_{t_{n}}, E_{+}\right)=0$ for all $m \in \mathbb{N}$.

Then $\left(x_{t_{n}}\right)$ is convergent.

Proof. By (i), if $m \in \mathbb{N}$ is fixed the sequence $\left(P_{t_{n}-t_{m}} x_{t_{m}}\right)_{n \in \mathbb{N}}$ is bounded. Assumption (ii) implies that there exist $z_{n} \in E, n \in \mathbb{N}$, such that

$$
0 \leq x_{t_{n}} \leq P_{t_{n}-t_{m}} x_{t_{m}}+z_{n} \text { and } \quad \lim _{n}\left\|z_{n}\right\|=0 \text {. }
$$

Then $\left(x_{t_{n}}\right)$ is also bounded. Let $\alpha:=\liminf _{n}\left\|x_{t_{n}}\right\|<\infty$. For fixed $\varepsilon>0$ choose $m \in \mathbb{N}$ such that $\left\|x_{t_{m}}\right\|<\alpha+\varepsilon$. Since $P_{t_{n}-t_{m}}$ is non-expansive $(*)$ yields

$$
\limsup _{n}\left\|x_{t_{n}}\right\| \leq \limsup _{n}\left\|P_{t_{n}-t_{m}} x_{t_{m}}\right\| \leq \alpha+\varepsilon .
$$

Thus $\limsup _{n}\left\|x_{t_{n}}\right\| \leq \alpha$, and hence $\alpha=\lim _{n}\left\|x_{t_{n}}\right\|$ exists.

Let $M:=\sup _{n}\left\|x_{t_{n}}\right\|<\infty$ and fix $\varepsilon>0$. Choose $\delta>0$ corresponding to $M$ and $\varepsilon$ as in (2.1). There exists $r \in \mathbb{N}$ such that

$$
\left|\left\|x_{t_{n}}\right\|-\left\|x_{t_{m}}\right\|\right|<\delta \quad \text { for } \quad n, m \geq r .
$$

Assumption (i) implies that there is $r_{1}>r$ such that

$$
\left\|P_{t_{n}-t_{r}} x_{t_{r}}-P_{t_{m}-t_{r}} x_{t_{r}}\right\|<\varepsilon \quad \text { for } \quad n, m \geq r_{1} .
$$

By assumption (ii), there exists $r_{2} \geq r_{1}$ such that

$$
d\left(P_{t_{n}-t_{r}} x_{t_{r}}-x_{t_{n}}, E_{+}\right)<\delta \text { for } n \geq r_{2} .
$$

In particular, we find $w_{n} \in E, n \geq r_{2}$, such that

$$
0 \leq x_{t_{n}} \leq P_{t_{n}-t_{r}} x_{t_{r}}+w_{n} \text { and }\left\|w_{n}\right\|<\delta .
$$


Then

$$
\left\|x_{t_{n}}\right\|-\delta<\left\|P_{t_{n}-t_{r}} x_{t_{r}}\right\| \leq\left\|x_{t_{r}}\right\|<\left\|x_{t_{n}}\right\|+\delta, \quad n \geq r_{2},
$$

and hence

$$
\left|\left\|P_{t_{n}-t_{r}} x_{t_{r}}\right\|-\left\|x_{t_{n}}\right\|\right|<\delta \quad \text { for } \quad n \geq r_{2} .
$$

From (2.1) we obtain

$$
\left\|P_{t_{n}-t_{r}} x_{t_{r}}-x_{t_{n}}\right\|<\varepsilon \quad \text { for } n \geq r_{2} .
$$

Thus, if $n, m \geq r_{2}$, then

$$
\begin{aligned}
\left\|x_{t_{n}}-x_{t_{m}}\right\| & \leq\left\|x_{t_{n}}-P_{t_{n}-t_{r}} x_{t_{r}}\right\|+\left\|P_{t_{n}-t_{r}} x_{t_{r}}-P_{t_{m}-t_{r}} x_{t_{r}}\right\|+\left\|P_{t_{m}-t_{r}} x_{t_{r}}-x_{t_{m}}\right\| \\
& <3 \varepsilon .
\end{aligned}
$$

Hence $\left(x_{t_{n}}\right)$ is a Cauchy sequence and the assertion follows.

Remark 2.5. If in Lemma 2.4 we replace assumption (i) by

$$
\left\{P_{t_{n}-t_{m}} x_{t_{m}}: n \in \mathbb{N}\right\} \text { is relatively compact for all } m \in \mathbb{N} \text {, }
$$

then there is a subsequence $\left(x_{s_{n}}\right)$ of $\left(x_{t_{n}}\right)$ which is convergent. In fact, by a diagonal sequence argument we can choose a subsequence $\left(s_{n}\right)$ of $\left(t_{n}\right)$ such that $\left(P_{s_{n}-t_{m}} x_{t_{m}}\right)_{n \in \mathbb{N}}$ is convergent for each $m \in \mathbb{N}$. Thus $\left(P_{s_{n}-s_{m}} x_{s_{m}}\right)_{n \in \mathbb{N}}$ converges for each $m \in \mathbb{N}$. Moreover, $\lim _{n} d\left(P_{s_{n}-s_{m}} x_{s_{m}}-x_{s_{n}}, E_{+}\right)=0$ for all $m \in \mathbb{N}$. Now Lemma 2.4 with $\left(t_{n}\right)$ replaced by $\left(s_{n}\right)$ yields the assertion.

\section{STABILITY AND ASYMPTOTIC COMPACTNESS OF ASYMPTOTICALLY DOMINATED REPRESENTATIONS}

As in the previous section $E$ denotes an ordered Banach space with a closed, generating, normal positive cone $E_{+}$, and we assume that the norm is monotone on $E_{+}$. Throughout the whole paper $J$ stands for the additive semigroup $\mathbb{N}$ and $\mathbb{R}_{+}$, respectively. If $D \subseteq E$, then every homomorphism $\mathcal{S}: t \mapsto S_{t}$ from $J$ into the semigroup $D^{D}$ of all mappings from $D$ into itself is called a representation of $J$ in $D^{D}$. We also write $\mathcal{S}=\left(S_{t}\right)_{t \in J}$ for short. If not stated explicitly we do not impose any continuity conditions on $\mathcal{S}$. For $J=\mathbb{N}$ each representation is given by the sequence of all powers $\left(S^{n}\right)_{n \in \mathbb{N}}$ of $S=S_{1}$.

Let $D \subseteq E_{+}$. We say that the representation $\mathcal{S}=\left(S_{t}\right)_{t \in J}$ in $D^{D}$ is asymptotically dominated by the representation $\mathcal{T}=\left(T_{t}\right)_{t \in J}$ in $D^{D}$ if

$$
\lim _{t \rightarrow \infty} d\left(T_{t} x-S_{t} x, E_{+}\right)=0 \text { for all } x \in D .
$$

For a mapping $P \in \bar{D}^{D}$ the representation $\mathcal{S}=\left(S_{t}\right)_{t \in J}$ in $D^{D}$ is called asymptotically dominated by $P$ if

$$
\lim _{t \rightarrow \infty} d\left(P x-S_{t} x, E_{+}\right)=0 \text { for all } x \in D .
$$

Our aim is to study asymptotic properties of asymptotically dominated representations. The representation $\mathcal{S}$ is called strongly stable if $\lim _{t \rightarrow \infty} S_{t} x$ exists for all $x \in D$, and $\mathcal{S}$ is called strongly asymptotically compact if for every $x \in D$ and every sequence $\left(t_{n}\right)$ in $J$ converging to $\infty$ there is a subsequence $\left(s_{n}\right)$ of $\left(t_{n}\right)$ such that $\lim _{n} S_{s_{n}} x$ exists in $E$. If the representation $\mathcal{S}$ is strongly continuous, i.e., $t \mapsto S_{t} x$ is continuous for all $x \in D$, then $\mathcal{S}$ is strongly asymptotically compact if and only if each orbit $\left\{S_{t} x: t \in J\right\}, x \in D$, is relatively compact in $E$. Note that a strongly 
continuous representation in $\mathcal{L}(E)$ which is strongly asymptotically compact is also called almost periodic (see [8] p.142]).

First we discuss inheritance of strong stability for asymptotically dominated representations.

Theorem 3.1. Let $E$ be a uniformly order convex Banach space and let $D \subseteq$ $E_{+}$. Assume that $\mathcal{S}=\left(S_{t}\right)_{t \in J}$ is a representation in $D^{D}$ which is asymptotically dominated either:

a) by a non-expansive mapping $P \in \bar{D}^{D}$ or

b) by a strongly stable representation $\mathcal{T}=\left(T_{t}\right)_{t \in J}$ of non-expansive mappings in $D^{D}$.

Then $\mathcal{S}=\left(S_{t}\right)_{t \in J}$ is strongly stable.

Proof. a) Let $x \in D$ and let $\left(t_{n}\right)$ be a sequence in $J$ such that $\lim _{n} t_{n}=\infty$. For $t \in J$ set $x_{t}:=S_{t} x$ and $P_{t}:=P$. Then $\left(x_{t}\right)_{t \in J},\left(P_{t}\right)_{t \in J}$ and $\left(t_{n}\right)$ satisfy the assumptions of Lemma 2.4, and hence $\left(x_{t_{n}}\right)$ is convergent. Since this is true for every sequence $\left(t_{n}\right)$ in $J$ converging to $\infty$ the stability of $\mathcal{S}$ follows.

b) Let $P x:=\lim _{t \rightarrow \infty} T_{t} x, x \in D$. Then $P \in \bar{D}^{D}$ is non-expansive and $\mathcal{S}$ is asymptotically dominated by $P$. Now the assertion follows from a).

An operator $T \in \mathcal{L}(E)$ is called positive if $T E_{+} \subseteq E_{+}$. Clearly, the set $\mathcal{L}(E)_{+}$ of all positive operators on $E$ can be identified with a subsemigroup of $E_{+}^{E_{+}}$. Thus from Theorem 3.1 we can deduce the following result on representations in $\mathcal{L}(E)_{+}$.

Corollary 3.2. Let $E$ be a uniformly order convex Banach space. Assume that $\mathcal{S}=\left(S_{t}\right)_{t \in J}$ is a representation in $\mathcal{L}(E)_{+}$which is asymptotically dominated either.

a) by a contraction $P \in \mathcal{L}(E)_{+}$or

b) by a strongly stable representation $\mathcal{T}=\left(T_{t}\right)_{t \in J}$ of contractions in $\mathcal{L}(E)_{+}$.

Then $\mathcal{S}=\left(S_{t}\right)_{t \in J}$ is strongly stable.

We now discuss the inheritance of strong asymptotic compactness under asymptotic domination. We obtain the following analogue of Theorem 3.1.

Theorem 3.3. Let $E$ be a uniformly order convex Banach space and let $D \subseteq$ $E_{+}$. Assume that $\mathcal{S}=\left(S_{t}\right)_{t \in J}$ is a representation in $D^{D}$ which is asymptotically dominated by a strongly asymptotically compact representation $\mathcal{T}=\left(T_{t}\right)_{t \in J}$ of nonexpansive mappings in $D^{D}$. Then $\mathcal{S}=\left(S_{t}\right)_{t \in J}$ is strongly asymptotically compact.

Proof. Let $x \in D$ and let $\left(t_{n}\right)$ be a sequence in $J$ such that $\lim _{n} t_{n}=\infty$. Set $x_{t}:=S_{t} x$ and $P_{t}:=T_{t}, t \in J$. Our assumptions imply that $\left(x_{t}\right)_{t \in J},\left(P_{t}\right)_{t \in J}$ and $\left(t_{n}\right)$ fulfill the conditions of Remark 2.5. Thus $\left(S_{t_{n}} x\right)$ has a convergent subsequence, and hence $\mathcal{S}$ is strongly asymptotically compact.

For representations in $\mathcal{L}(E)_{+}$the previous result reads as follows.

Corollary 3.4. Let $E$ be a uniformly order convex Banach space. Assume that $\mathcal{S}=$ $\left(S_{t}\right)_{t \in J}$ and $\mathcal{T}=\left(T_{t}\right)_{t \in J}$ are representations in $\mathcal{L}(E)_{+}$such that $\mathcal{S}$ is asymptotically dominated by $\mathcal{T}$, and $\mathcal{T}$ is contractive and strongly asymptotically compact. Then $\mathcal{S}=\left(S_{t}\right)_{t \in J}$ is strongly asymptotically compact.

The following example shows that in the results of this section the uniform order convexity of the space cannot be omitted. 
Example 3.5. Let $E=c$ be the space of convergent sequences endowed with the sup-norm. Let $T=I d$ and define $S \in \mathcal{L}(E)_{+}$by $S\left(\xi_{n}\right):=\left(\frac{n}{n+1} \xi_{n}\right)$. Then $\left(T^{n}\right)_{n \in \mathbb{N}}$ is strongly stable and contractive, and $\left(S^{n}\right)$ is (strongly asymptotically) dominated by $\left(T^{n}\right)$. However, $\left(S^{n}\right)$ is not even strongly asymptotically compact.

\section{Stability AND ALmost PERIODiCity OF ASYMPtotically DOMinAted REPRESENTATIONS ON BANACH LATTICES}

In this section we assume that $E$ is a Banach lattice and investigate asymptotic properties of asymptotically dominated representations in $\mathcal{L}(E)_{+}$. We shall see that, compared to the previous section, the uniform order convexity of the space $E$ can be replaced by a considerably weaker condition.

Note that in a Banach lattice $E$ we always have $d\left(x, E_{+}\right)=\left\|(-x)_{+}\right\|, x \in E$, and hence a representation $\mathcal{S}=\left(S_{t}\right)_{t \in J}$ in $\mathcal{L}(E)_{+}$is asymptotically dominated by a representation $\mathcal{T}=\left(T_{t}\right)_{t \in J}$ in $\mathcal{L}(E)_{+}$if and only if

$$
\lim _{t \rightarrow \infty}\left\|\left(S_{t} x-T_{t} x\right)_{+}\right\|=0 \quad \text { for all } \quad x \in E_{+} .
$$

We recall the Glicksberg-deLeeuw Decomposition Theorem (see [6, Section 2.4], [8, p.150]) which will play an important role in the proof of the main results of this section. By $\Gamma$ we denote the unit circle $\{\lambda \in \mathbb{C}:|\lambda|=1\}$.

Glicksberg-deLeeuw Decomposition Theorem 4.1. Let $F$ be a Banach space and let $\mathcal{T}=\left(T_{t}\right)_{t \in J}$ be a strongly continuous almost periodic representation in $\mathcal{L}(F)$. Then $F$ can be decomposed into a direct sum $F=F_{0}(\mathcal{T}) \oplus F_{r}(\mathcal{T})$ where

$$
\begin{gathered}
F_{0}(\mathcal{T})=\left\{x \in F: \lim _{t \rightarrow \infty}\left\|T_{t} x\right\|=0\right\} \quad \text { and } \\
F_{r}(\mathcal{T})=\overline{\operatorname{lin}}\left\{x \in F: \text { there is a character } \alpha: J \rightarrow \Gamma \text { such that } T_{t} x=\alpha(t) x\right\} .
\end{gathered}
$$

Moreover, the projection $Q_{\mathcal{T}}$ of $F$ onto $F_{r}(\mathcal{T})$ with kernel $F_{0}(\mathcal{T})$ is an accumulation point of $\left(T_{t}\right)_{t \in J}$ with respect to the strong operator topology.

We need the following construction which can be found e.g. in [20, II.8, Example 1]. Let $x^{\prime} \in E_{+}^{\prime}$ be a positive linear form on the Banach lattice $E$. Then $N\left(x^{\prime}\right):=$ $\left\{x \in E:\left\langle x^{\prime},|x|\right\rangle=0\right\}$ is a closed ideal in $E$, and $\left\|x+N\left(x^{\prime}\right)\right\|:=\left\langle x^{\prime},|x|\right\rangle, x \in E$, defines a lattice norm on the quotient space $E / N\left(x^{\prime}\right)$. The completion $\left(E, x^{\prime}\right)$ of $E / N\left(x^{\prime}\right)$ with respect to this norm is a Banach lattice. The norm is additive on $\left(E, x^{\prime}\right)_{+}$, and hence the space $\left(E, x^{\prime}\right)$ is uniformly order convex (see Example $2.2 \mathrm{~b})$ ). The quotient map $q: E \rightarrow E / N\left(x^{\prime}\right)$ defines a lattice homomorphism $j_{x^{\prime}}: E \rightarrow\left(E, x^{\prime}\right)$. If $T \in \mathcal{L}(E)_{+}$is an operator such that $T^{\prime} x^{\prime} \leq x^{\prime}$, then the ideal $N\left(x^{\prime}\right)$ is $T$-invariant, and $T$ induces a positive contraction $\tilde{T}$ on $\left(E, x^{\prime}\right)$ given by $\tilde{T} j_{x^{\prime}} x=j_{x^{\prime}} T x, x \in E$.

Recall that a Banach lattice $E$ has order continuous norm if whenever $\left(x_{\alpha}\right)$ is a decreasing net in $E_{+}$with $\inf _{\alpha} x_{\alpha}=0$, then $\lim _{\alpha}\left\|x_{\alpha}\right\|=0$. From Proposition 2.3 it follows that every uniformly order convex Banach lattice has order continuous norm (see [20, II.5.10, II.5.15]). A set $A$ in the Banach lattice $E$ is called almost order bounded if for every $\varepsilon>0$ there exists $x_{\varepsilon} \in E_{+}$such that $\left\|\left(|x|-x_{\varepsilon}\right)_{+}\right\| \leq \varepsilon$ for all $x \in A$. A linear form $x^{\prime} \in E_{+}^{\prime}$ is said to be strictly positive if $\left\langle x^{\prime}, x\right\rangle>0$ for all $x \in E_{+} \backslash\{0\}$. The following lemma connects convergence of an almost order bounded sequence in $E$ with convergence in $\left(E, x^{\prime}\right)$ (see [11, 2.4.8], [15] Lemma $3.8])$. 
Lemma 4.2. Let $\left(x_{n}\right)$ be an almost order bounded sequence in a Banach lattice $E$ with order continuous norm, and let $x^{\prime}$ be a strictly positive linear form on $E$. Then $\left(x_{n}\right)$ is convergent in $E$ if and only if $\left(j_{x^{\prime}} x_{n}\right)$ is convergent in $\left(E, x^{\prime}\right)$.

We come to the main results of this section in which we discuss the inheritance of strong stability and almost periodicity of asymptotically dominated representations in $\mathcal{L}(E)_{+}$.

Theorem 4.3. Let $E$ be a Banach lattice with order continuous norm and let $\mathcal{S}=$ $\left(S_{t}\right)_{t \in J}$ and $\mathcal{T}=\left(T_{t}\right)_{t \in J}$ be strongly continuous representations in $\mathcal{L}(E)_{+}$such that $\mathcal{S}$ is asymptotically dominated by $\mathcal{T}$. If $\mathcal{T}$ is almost periodic, then $\mathcal{S}$ is almost periodic.

Proof. A) First we consider the case $J=\mathbb{N}$. By passing to an equivalent norm we may assume that $\mathcal{S}$ consists of contractions. Now fix $x \in E_{+}$. Let $F$ be the closed ideal in $E$ generated by $M=\left\{S_{m_{k}} T_{n_{k}} \ldots S_{m_{0}} T_{n_{0}} x: m_{0}, n_{0}, \ldots, m_{k}, n_{k} \in\right.$ $\mathbb{N}, k \in \mathbb{N}\}$. Then $F$ is invariant for $\mathcal{S}$ and $\mathcal{T}$, and $u=\sum_{n \in \mathbb{N}} 2^{-n} x_{n}$ is a weak order unit of $F$, where $\left\{x_{n}: n \in \mathbb{N}\right\}$ is an enumeration of $M$. Thus, by restricting $\mathcal{S}$ and $\mathcal{T}$ to $F$ we may assume that $E$ contains a weak order unit. By [7, 1.b.15] there is a strictly positive linear form $z^{\prime} \in E_{+}^{\prime}$. Since $\mathcal{T}$ is almost periodic $\mathcal{T}$ is mean ergodic with ergodic projection $P_{\mathcal{T}}$ (see [8, p.164]). Consider the closed ideal $I:=\left\{z \in E: \quad P_{\mathcal{T}}|z|=0\right\}$. The order continuity of the norm implies that $I$ is a projection band (see [11, 2.4.4]); hence $E=I \oplus I^{\perp}$ where $I^{\perp}=\{z \in E:|z| \wedge|y|=0$ for all $y \in I\}$. Let $R$ be the band projection from $E$ onto $I^{\perp}$. Note that $0 \leq R \leq I d$ and $R$ is a lattice homomorphism (see [20, II.2.9]). We claim that $\left\{S_{n} z: n \in \mathbb{N}\right\}$ is relatively compact in $E$ for every $z \in E_{+}$. It suffices to show that this is true for $z \in I_{+}$and $z \in\left(I^{\perp}\right)_{+}$, respectively.

1st case: $z \in I_{+}$. Since $\mathcal{T}$ commutes with the ergodic projection $P_{\mathcal{T}}$ the ideal $I$ is $\mathcal{T}$-invariant. Denote by $\mathcal{T}$ the restriction of $\mathcal{T}$ to $I$. Let $w \in I$ and $\alpha: \mathbb{N} \rightarrow \Gamma$ be a character such that $T_{n} w=\alpha(n) w, n \in \mathbb{N}$. Then $|w| \leq T_{n}|w|, n \in \mathbb{N}$, and hence $|w| \leq P_{\mathcal{T}}|w|=0$ which implies $w=0$. Since $\mathcal{T}_{\mid}$is almost periodic it follows from the Glicksberg-deLeeuw Decomposition Theorem that $\mathcal{T}$ converges strongly to 0 . In particular, $\lim _{n} T_{n} z=0$. Since $\mathcal{S}$ is asymptotically dominated by $\mathcal{T}$ we obtain $\lim _{n} S_{n} z=0$.

2nd case: $z \in\left(I^{\perp}\right)_{+}$. By assumption $\left\{T_{n} z: n \in \mathbb{N}\right\}$ is relatively compact, and hence almost order bounded. Since $\mathcal{S}$ is asymptotically dominated by $\mathcal{T}$ the set $\left\{S_{n} z: n \in \mathbb{N}\right\}$ is almost order bounded. Let $x^{\prime}:=P_{\mathcal{T}}^{\prime} z^{\prime}$. Then $T_{n}^{\prime} x^{\prime}=$ $x^{\prime}, n \in \mathbb{N}$. The induced representation $\tilde{\mathcal{T}}=\left(\tilde{T}_{n}\right)_{n \in \mathbb{N}}$ on $\left(E, x^{\prime}\right)$ determined by $\tilde{T}_{n} j_{x^{\prime}}=j_{x^{\prime}} T_{n}, n \in \mathbb{N}$, is positive, contractive and almost periodic. Moreover,

$$
\begin{aligned}
\lim _{n} d\left(\tilde{T}_{n-m}\left(j_{x^{\prime}} S_{m} z\right)-j_{x^{\prime}} S_{n} z,\left(E, x^{\prime}\right)_{+}\right) & =\lim _{n}\left\|\left[j_{x^{\prime}} S_{n} z-j_{x^{\prime}} T_{n-m} S_{m} z\right]_{+}\right\| \\
& =\lim _{n}\left\langle x^{\prime},\left(S_{n} z-T_{n-m} S_{m} z\right)_{+}\right\rangle=0 .
\end{aligned}
$$

Remark 2.5 applied to the sequence $\left(j_{x^{\prime}} S_{n} z\right) \subseteq\left(E, x^{\prime}\right)_{+}$, the operators $\tilde{T}_{n} \in$ $\mathcal{L}\left(\left(E, x^{\prime}\right)\right)_{+}$and every sequence $\left(t_{n}\right)$ in $\mathbb{N}$ converging to $\infty$ shows that $\left\{j_{x^{\prime}} S_{n} z\right.$ : $n \in \mathbb{N}\}$ is relatively compact in $\left(E, x^{\prime}\right)$. From

$$
\begin{aligned}
& \left\|j_{x^{\prime}} R S_{n} z-j_{x^{\prime}} R S_{m} z\right\|_{\left(E, x^{\prime}\right)}=\left\langle x^{\prime},\left|R S_{n} z-R S_{m} z\right|\right\rangle \\
& \quad \leq\left\langle x^{\prime},\left|S_{n} z-S_{m} z\right|\right\rangle=\left\|j_{x^{\prime}} S_{n} z-j_{x^{\prime}} S_{m} z\right\|_{\left(E, x^{\prime}\right)}, \quad n, m \in \mathbb{N},
\end{aligned}
$$


we derive that $\left\{j_{x^{\prime}} R S_{n} z: n \in \mathbb{N}\right\}$ is relatively compact in $\left(E, x^{\prime}\right)$. Since $\left.x^{\prime}\right|_{I^{\perp}}$ is strictly positive and $\left\{R S_{n} z: n \in \mathbb{N}\right\}$ is almost order bounded Lemma 4.2 implies that $\left\{R S_{n} z: n \in \mathbb{N}\right\}$ is relatively compact in $I^{\perp} \subseteq E$.

Now we show that $\left\{S_{n} z: n \in \mathbb{N}\right\}$ is relatively compact. Fix $\varepsilon>0$. Since $\left\{S_{n} z: n \in \mathbb{N}\right\}$ is almost order bounded there exists $y \in E_{+}$such that

$$
\left\|\left(\left|S_{k} z-S_{l} z\right|-y\right)_{+}\right\| \leq \varepsilon \quad \text { for } \quad k, l \in \mathbb{N} .
$$

By the contractivity of the operators $S_{n}$ and $I d-R$ we obtain

$$
\begin{aligned}
\left\|S_{n}\left(S_{k} z-S_{l} z\right)\right\|-\left\|R\left(S_{k} z-S_{l} z\right)\right\| & \leq\left\|S_{n}\left(S_{k} z-S_{l} z\right)\right\|-\left\|S_{n} R\left(S_{k} z-S_{l} z\right)\right\| \\
& \leq\left\|S_{n}(I d-R)\left|S_{k} z-S_{l} z\right|\right\| \\
& \leq\left\|S_{n}(I d-R) y\right\|+\varepsilon .
\end{aligned}
$$

From the first case we know that $\lim _{n} S_{n}(I d-R) y=0$. Hence there exists $n_{0} \in \mathbb{N}$ such that

$$
\left\|S_{n_{0}}\left(S_{k} z-S_{l} z\right)\right\| \leq\left\|R\left(S_{k} z-S_{l} z\right)\right\|+2 \varepsilon \quad \text { for } \quad k, l \in \mathbb{N} .
$$

Since $\left\{R S_{n} z: n \in \mathbb{N}\right\}$ is relatively compact there exists $m_{0} \in \mathbb{N}$ such that

$$
\left\{R S_{n} z: n \in \mathbb{N}\right\} \subseteq\left\{R S_{m} z: 0 \leq m \leq m_{0}\right\}+\varepsilon B_{E} .
$$

Now let $n>m_{0}+n_{0}$. There exists $0 \leq m \leq m_{0}$ such that

$$
\left\|R S_{n-n_{0}} z-R S_{m} z\right\| \leq \varepsilon \text {. }
$$

By $(*)$ we have

$$
\left\|S_{n} z-S_{m+n_{0}} z\right\| \leq\left\|R S_{n-n_{0}} z-R S_{m} z\right\|+2 \varepsilon \leq 3 \varepsilon .
$$

Thus $\left\{S_{n} z: n \in \mathbb{N}\right\} \subseteq\left\{S_{m} z: 0 \leq m \leq m_{0}+n_{0}\right\}+3 \varepsilon B_{E}$, i.e., $\left\{S_{n} z: n \in \mathbb{N}\right\}$ is totally bounded and hence relatively compact. This proves the assertion for $J=\mathbb{N}$.

B) If $J=\mathbb{R}_{+}$, then from A) it follows that $\left\{S_{n} x: n \in \mathbb{N}\right\}$ is relatively compact for each $x \in E$. The strong continuity of $\mathcal{S}$ implies that $\left\{S_{t} x: 0 \leq t \leq 1\right\}$ is compact. Hence $\left\{S_{t} x: t \geq 0\right\}=\left\{S_{t} x: 0 \leq t \leq 1\right\}+\left\{S_{t} S_{n} x: t \in[0,1], n \in \mathbb{N}\right\}$ is relatively compact for each $x \in E$.

Remark 4.4. The proof shows that the strong continuity of $\mathcal{T}$ is not needed and that instead of the almost periodicity it suffices to require only asymptotic compactness of $\mathcal{T}$.

From Theorem 4.3 we can deduce the following result on the inheritance of strong stability.

Theorem 4.5. Let $E$ be a Banach lattice with order continuous norm and let $\mathcal{S}=$ $\left(S_{t}\right)_{t \in J}$ and $\mathcal{T}=\left(T_{t}\right)_{t \in J}$ be strongly continuous representations in $\mathcal{L}(E)_{+}$such that $\mathcal{S}$ is asymptotically dominated by $\mathcal{T}$. If $\mathcal{T}$ is strongly stable, then $\mathcal{S}$ is strongly stable.

Proof. Theorem 4.3 implies that $\mathcal{S}$ is almost periodic. Since $\mathcal{S}$ is asymptotically dominated by $\mathcal{T}$ and $\mathcal{T}$ is strongly stable the corresponding Glicksberg-deLeeuw projections $Q_{\mathcal{S}}$ and $Q_{\mathcal{T}}$ satisfy

$$
0 \leq S_{t} Q_{\mathcal{S}} \leq T_{t} Q_{\mathcal{T}}=Q_{\mathcal{T}}, \quad t \in J
$$

By [17, Theorem 1.4] the following inclusions for the spectra hold

$$
\sigma\left(S_{t} Q_{\mathcal{S}}\right) \cap \Gamma \subseteq \sigma\left(Q_{\mathcal{T}}\right) \cap \Gamma \subseteq\{1\}, \quad t \in J .
$$


Now the Glicksberg-deLeeuw Decomposition Theorem yields $\left.S_{t}\right|_{E_{r}(\mathcal{S})}=I d_{E_{r}(\mathcal{S})}$ and $\lim _{t \rightarrow \infty} S_{t} x=0$ for $x \in E_{0}(\mathcal{S})$. Hence $\mathcal{S}$ is strongly stable.

Remark 4.6. From [13, Proposition 3.1 and 3.4] it follows that in order to have inheritance of almost periodicity and stability as formulated in Theorem 4.3 and 4.5, respectively, the order continuity of the norm of $E$ is also necessary at least for Banach lattices which contain a topological orthogonal system or which are countably order complete.

The following examples show that positivity of the representation $\mathcal{S}$ cannot be omitted. We point out that in the second example the operators belonging to $\mathcal{S}$ are even regular, i.e., the difference of two positive operators.

Example 4.7. a) Consider on $L^{p}(\Gamma), 1 \leq p<\infty$, the operator $S$ defined by $S f(z)=z f(z), f \in L^{p}(\Gamma), z \in \Gamma$. Then $\mathcal{S}=\left(S^{n}\right)_{n \in \mathbb{N}}$ is dominated by the trivial representation $\mathcal{T}=(I d)_{n \in \mathbb{N}}$ in the following sense:

$$
\left|S^{n} f\right|=|f|, \quad f \in L^{p}(\Gamma) .
$$

Note that $S$ is an isometry and has no eigenvalues. By the Glicksberg-deLeeuw Decomposition Theorem $\mathcal{S}$ cannot be almost periodic whereas $\mathcal{T}$ is even strongly stable.

b) Let $\alpha \in \mathbb{R} \backslash \mathbb{Q}$. Define $S$ on $L^{p}(\Gamma), 1 \leq p<\infty$, by $(S f)(z)=h(z) f\left(e^{i \alpha \pi} z\right)$ where $h(z)=1$ if $\operatorname{Re} z \geq 0$ and $h(z)=-1$ if $\operatorname{Re} z<0$. The modulus of the operator $S$ exists and is given by $(|S| f)(z)=f\left(e^{i \alpha \pi} z\right)$. The representation $\mathcal{T}=\left(|S|^{n}\right)_{n \in \mathbb{N}}$ is almost periodic, and the representation $\mathcal{S}=\left(S^{n}\right)_{n \in \mathbb{N}}$ is dominated by $\mathcal{T}$ in the following sense:

$$
\left|S^{n} f\right| \leq|S|^{n}|f|, \quad f \in L^{p}(\Gamma) .
$$

On the other hand $\left\{S^{n} \mathbf{1}: n \in \mathbb{N}\right\}$ is not relatively compact in $L^{p}(\Gamma)$ where $\mathbf{1}$ denotes the constant function 1 . Hence $\mathcal{S}$ is not almost periodic.

\section{REFERENCES}

1. C.D. Aliprantis and O. Burkinshaw, Positive Operators, Academic Press, Orlando, London, 1985. MR 87h:47086

2. F. Andreu and J.M. Mazon, On the boundary spectrum of dominated $C_{0}$-semigroups, Semigroup Forum 38 (1989), 129-139. MR 90a:47095

3. W. Arendt and C.J.K. Batty, Domination and ergodicity for positive semigroups, Proc. Amer. Math. Soc. 114 (1992), 743-747. MR 92f:47038

4. C.J.K. Batty and D.W. Robinson, Positive one-parameter semigroups on ordered Banach spaces, Acta Applicandae 2 (1984), 221-296. MR 86b:47068

5. V. Caselles, On the peripheral spectrum of positive operators, Isr. J. Math. 58 (1987), 144-160. MR 88j:47054

6. U. Krengel, Ergodic Theorems, De Gruyter, Berlin, New York, 1985. MR 87i:28001

7. J. Lindenstrauss and L. Tzafriri, Classical Banach Spaces II, Springer-Verlag, Berlin, Heidelberg, New York, 1979. MR 81c:46001

8. Yu.I. Lyubich, Introduction to the Theory of Banach Representations of Groups, Birkhäuser, Basel, Boston, Berlin, 1988. MR 90i:22001

9. J. Martinez, The essential spectral radius of dominated positive operators, Proc. Amer. Math. Soc. 118 (1993), 489-492. MR 93g:47047

10. J. Martinez and J.M. Mazon, Quasi-compactness of dominated positive operators and $C_{0}-$ semigroups, Math. Z. 207 (1991), 109-120. MR 92g:47048

11. P. Meyer-Nieberg, Banach Lattices, Springer-Verlag, Berlin, Heidelberg, New York, 1991. MR 93f:46025 
12. B. de Pagter and A. Schep, Measures of non-compactness of operators in Banach lattices, J. Funct. Anal. 78 (1988), 31-55. MR 89d:47079

13. F. Räbiger, Ergodic Banach lattices, Indag. Math., New Series 1 (1990), 483-488. MR 92e: 46043

14. F. Räbiger, Stability and ergodicity of dominated semigroups: I. The uniform case, Math. Z. 214 (1993), 43-54. MR 94i:47066

15. F. Räbiger, Stability and ergodicity of dominated semigroups: II. The strong case, Math. Ann. 297 (1993), 103-116. MR 94k:47014

16. F. Räbiger, Attractors and asymptotic periodicity of positive operators on Banach lattices, Forum Math. 7 (1995), 665-683. MR 96k:47066

17. F. Räbiger and M.P.H. Wolff, Spectral and asymptotic properties of dominated operators, J. Austral. Math. Soc. (Series A) 63 (1997), 16-31. MR 98k:47073

18. F. Räbiger and M.P.H. Wolff, On the approximation of positive operators and the behaviour of the spectra of the approximants, Integr. Equ. Oper. Theory 28 (1997), 72-86. MR 98k:47074

19. F. Räbiger and M.P.H. Wolff, Spectral and asymptotic properties of resolvent-dominated operators, J. Austral. Math. Soc. Ser. A 68 (2000), 181-201. CMP 2000:08

20. H.H. Schaefer, Banach Lattices and Positive Operators, Springer-Verlag, New York, Heidelberg, Berlin, 1974. MR 54:11023

21. Yau-Chuen Wong and Kung-Fu Ng, Partially Ordered Toplogical Vector Spaces, Clarendon Press, Oxford, 1973. MR 56:12830

22. A.C. Zaanen, Riesz Spaces II, North-Holland, Amsterdam, 1983. MR 86b:46001

Sobolev Institute of Mathematics at Novosibirsk, Universitetskil PR.4, 630090 NovosiBIRSK, RUSSIA

E-mail address: emelanov@math.nsc.ru

Mathematisches Institut, Universität Tübingen, Auf der Morgenstelle 10, D-72076

Tübingen, Germany

E-mail address: utko@michelangelo.mathematik.uni-tuebingen.de

Mathematisches Institut, Universität Tübingen, Auf Der Morgenstelle 10, D-72076 TüBingen, Germany

E-mail address: frra@michelangelo.mathematik.uni-tuebingen.de

Mathematisches Institut, Universität Tübingen, Auf der Morgenstelle 10, D-72076 Tübingen, Germany

E-mail address: manfred.wolff@uni-tuebingen.de 